\title{
Hospitalization of Adolescents Aged 12-17 Years with Laboratory-Confirmed COVID-19 - COVID-NET, 14 States, March 1, 2020-April 24, 2021
}

\begin{abstract}
Fiona P. Havers, $\mathrm{MD}^{1}$; Michael Whitaker, $\mathrm{MPH}^{1}$; Julie L. Self, $\mathrm{PhD}^{1}$; Shua J. Chai, $\mathrm{MD}^{2,3}$; Pam Daily Kirley, $\mathrm{MPH}^{2}$; Nisha B. Alden, MPH ${ }^{4}$; Breanna Kawasaki, MPH ${ }^{4}$; James Meek, MPH ${ }^{5}$; Kimberly Yousey-Hindes, MPH ${ }^{5}$; Evan J. Anderson, MD ${ }^{6,7,8}$; Kyle P. Openo, DrPH ${ }^{7,9}$; Andrew Weigel, MSW ${ }^{10}$; Kenzie Teno, $\mathrm{MPH}^{10}$; Maya L. Monroe, MPH ${ }^{11}$; Patricia A. Ryan, MS ${ }^{11}$; Libby Reeg, MPH ${ }^{12}$; Alexander Kohrman, MPH ${ }^{12}$; Ruth Lynfield, MD ${ }^{13}$; Kathryn Como-Sabetti, $\mathrm{MPH}^{13}$; Mayvilynne Poblete, $\mathrm{MPH}^{14}$; Chelsea McMullen, MS ${ }^{15}$; Alison Muse, MPH ${ }^{16}$; Nancy Spina, MPH ${ }^{16}$; Nancy M. Bennett, MD ${ }^{17}$; Maria Gaitán ${ }^{17}$; Laurie M. Billing, MPH ${ }^{18}$; Jess Shiltz, MPH ${ }^{18}$; Melissa Sutton, MD ${ }^{19}$; Nasreen Abdullah, MD ${ }^{19}$; William Schaffner, MD ${ }^{20}$; H. Keipp Talbot, $\mathrm{MD}^{20}$; Melanie Crossland, $\mathrm{MPH}^{21}$; Andrea George, $\mathrm{MPH}^{21}$; Kadam Patel, $\mathrm{MPH}^{1,22}$; Huong Pham MPH ${ }^{1}$; Jennifer Milucky, MSPH ${ }^{1}$; Onika Anglin, MPH ${ }^{1,22}$; Dawud Ujamaa, MS ${ }^{1,22}$; Aron J. Hall, DVM ${ }^{1}$; Shikha Garg, MD ${ }^{1,23}$; Christopher A. Taylor, PhD ${ }^{1}$; COVID-NET Surveillance Team
\end{abstract}

\section{On June 4, 2021, this report was posted as an MMWR Early} Release on the MMWR website (https://www.cdc.gov/mmwr).

Most COVID-19-associated hospitalizations occur in older adults, but severe disease that requires hospitalization occurs in all age groups, including adolescents aged $12-17$ years (1). On May 10, 2021, the Food and Drug Administration expanded the Emergency Use Authorization for Pfizer-BioNTech COVID-19 vaccine to include persons aged $12-15$ years, and CDC's Advisory Committee on Immunization Practices recommended it for this age group on May 12,2021 . $^{*}$ Before that time, COVID-19 vaccines had been available only to persons aged $\geq 16$ years. Understanding and describing the epidemiology of COVID-19-associated hospitalizations in adolescents and comparing it with adolescent hospitalizations associated with other vaccine-preventable respiratory viruses, such as influenza, offers evidence of the benefits of expanding the recommended age range for vaccination and provides a baseline and context from which to assess vaccination impact. Using the Coronavirus Disease 2019-Associated Hospitalization Surveillance Network (COVID-NET), CDC examined COVID-19-associated hospitalizations among adolescents aged 12-17 years, including demographic and clinical characteristics of adolescents admitted during January 1-March 31, 2021, and hospitalization rates (hospitalizations per 100,000 persons) among adolescents during March 1, 2020-April 24, 2021. Among 204 adolescents who were likely hospitalized primarily for COVID-19 during January 1-March 31, 2021, 31.4\% were admitted to an intensive care unit (ICU), and $4.9 \%$ required invasive mechanical ventilation; there were no associated deaths. During March 1, 2020-April 24, 2021, weekly adolescent hospitalization rates peaked at 2.1 per 100,000 in early January 2021 , declined to 0.6 in mid-March, and then rose to 1.3 in April. Cumulative COVID-19-associated hospitalization rates during October 1, 2020-April 24, 2021, were 2.5-3.0 times higher than were influenza-associated hospitalization rates from three recent

\footnotetext{
*https://www.fda.gov/news-events/press-announcements/coronavirus-covid-19update-fda-authorizes-pfizer-biontech-covid-19-vaccine-emergency-use; https:// www.cdc.gov/media/releases/2021/s0512-advisory-committee-signing.html
}

influenza seasons (2017-18, 2018-19, and 2019-20) obtained from the Influenza Hospitalization Surveillance Network (FluSurv-NET). Recent increased COVID-19-associated hospitalization rates in March and April 2021 and the potential for severe disease in adolescents reinforce the importance of continued COVID-19 prevention measures, including vaccination and correct and consistent wearing of masks by persons not yet fully vaccinated or when required by laws, rules, or regulations. ${ }^{\dagger}$

COVID-NET is a population-based surveillance system of laboratory-confirmed COVID-19-associated hospitalizations in 99 counties across 14 states, ${ }^{\circledR}$ covering approximately $10 \%$ of the U.S. population. Included in surveillance are COVID-19-associated hospitalizations among residents in a predefined surveillance catchment area who had a positive realtime reverse transcription-polymerase chain reaction or rapid antigen detection test result for SARS-CoV-2 (the virus that causes COVID-19) during hospitalization or $\leq 14$ days before admission (2). Clinical and demographic data, updated monthly, were analyzed for adolescents aged 12-17 years hospitalized during January 1-March 31, 2021. Clinical and demographic characteristics were analyzed separately for patients whose primary reason for admission was likely COVID-19 and those whose primary reason for admission might not have been primarily related to COVID-19, despite receiving a positive SARS-CoV-2 laboratory test result. ${ }^{* *}$ Hospitalization rate data, updated weekly, were analyzed during March 1, 2020-April 24, 2021, to describe cumulative COVID-19-associated hospitalization rates in adolescents aged $12-17$ years and adults aged $\geq 18$ years and weekly COVID-19-associated hospitalization rates in children aged $0-4$ years and 5-11 years and adolescents aged $12-17$ years. In

\footnotetext{
${ }^{\dagger}$ https://www.cdc.gov/coronavirus/2019-ncov/vaccines/fully-vaccinated.html $\S$ California, Colorado, Connecticut, Georgia, Iowa, Maryland, Michigan, Minnesota, New Mexico, New York, Ohio, Oregon, Tennessee, and Utah.

https://www.cdc.gov/coronavirus/2019-ncov/covid-data/covid-net/purposemethods.html

** Those excluded were patients admitted for 1) labor and delivery (pregnant) (5.6\%), 2) inpatient procedures/surgery $(6.4 \%), 3)$ psychiatric reasons but requiring medical care $(20.2 \%)$, 4) trauma $(5.9 \%)$, and 5$)$ other or unknown reasons $(7.8 \%)$ with no recorded COVID-19-associated symptoms upon admission and who might have been identified through routine testing upon admission.
} 
addition, cumulative COVID-19-associated hospitalization rates among adolescents aged 12-17 years during October 1, 2020 April 24, 2021 (covering most of the typical October 1-April 30 season for influenza-associated hospitalization surveillance), were compared with influenza-associated hospitalization rates in the same age group across three influenza seasons (2017-18, 2018-19, and 2019-20) using data from FluSurv-NET ${ }^{\dagger \dagger}$ (3). Rate calculations are unadjusted and include all persons meeting the case definition (2). SAS statistical software (version 9.4; SAS Institute) was used for analyses. This activity was reviewed by $\mathrm{CDC}$ and was conducted consistent with applicable federal law and CDC policy. ${ }^{\$ \$}$

Among 376 adolescents hospitalized during January 1March 31, 2021, who received a positive SARS-CoV-2 laboratory test result, 172 (45.7\%) were analyzed separately because their primary reason for admission might not have been directly COVID-19-related (Table). Among the 204 patients who were likely admitted primarily for COVID-19-related illness, $52.5 \%$ were female, $31.4 \%$ were Hispanic or Latino (Hispanic), and 35.8\% were non-Hispanic Black. Overall, $70.6 \%$ had one or more underlying medical conditions, the most common of which were obesity $(35.8 \%)$, chronic lung disease, including asthma (30.9\%), and neurologic disorders (14.2\%); $31.4 \%$ of patients required ICU admission and $4.9 \%$ required invasive mechanical ventilation, but there were no associated deaths.

During March 1, 2020-April 24, 2021, the cumulative COVID-19-associated adolescent hospitalization rate (49.9) was 12.5 times lower than that in adults aged $\geq 18$ years (675.6). Weekly COVID-19-associated adolescent hospitalization rates (3-week moving average) were comparable to rates among those aged $0-4$ years, but higher than rates among children aged 5-11 years (Figure 1). Weekly adolescent hospitalization rates peaked at 2.1 per 100,000 during the week ending January 9, 2021, declined to 0.6 during the week ending March 13, 2021, then increased to 1.3 and 1.2 for the weeks ending April 17 and 24, 2021, respectively. Rates among adolescents in two of 14 sites (Maryland and Michigan) were highest during April 2021 compared with all other weeks within their respective sites since surveillance began on March 1, 2020. Cumulative COVID-19-associated hospitalization rates during October 1, 2020-April 24, 2021, were 2.5-3.0 times higher than seasonal influenza-associated hospitalization rates during three recent influenza seasons (October 1-April 30) (Figure 2).

\footnotetext{
$\dagger_{\dagger}$ FluSurv-NET, which has similar methods for case ascertainment and catchment areas as COVID-NET, conducts seasonal laboratory-confirmed influenzaassociated hospitalization surveillance during October 1-April 30 annually.

$\$ \$ 45$ C.F.R. part 46, 21 C.F.R. part 56; 42 U.S.C. Sect. 241(d); 5 U.S.C. Sect. 552a; 44 U.S.C. Sect. 3501 et seq.
}

\section{Discussion}

COVID-NET data indicate that COVID-19-associated hospitalization rates were lower in adolescents aged $12-17$ years compared with those in adults but exceeded those among children aged 5-11 years during March 1, 2020-April 24, 2021. Moreover, COVID-19-associated hospitalization rates among adolescents increased during March-April 2021, and nearly one third of 204 recently hospitalized adolescents required ICU admission. Rates of COVID-19-associated hospitalization among adolescents also exceeded historical rates of seasonal influenza-associated hospitalization during comparable periods. Recent increased hospitalization rates and the potential for severe disease reinforce the importance of continued COVID-19 prevention measures among adolescents, including vaccination and correct and consistent wearing of masks.

After declines in January and February 2021, weekly population-based rates of COVID-19-associated hospitalization among adolescents increased during March and April, and in two COVID-NET sites (Maryland and Michigan) the highest adolescent COVID-19-associated hospitalization rates in their respective sites since the start of the COVID-19 pandemic occurred during this period. This trend contrasts with hospitalization rates among persons aged $\geq 65$ years, the group with the highest COVID-19 vaccination coverage, among whom hospitalization rates in COVID-NET stabilized during the same period.99 Increased hospitalization rates among adolescents might be related, in part, to circulation of particularly transmissible SARS-CoV-2 variants, ${ }^{* * *}$ the larger numbers of children returning to school or other in-person indoor activities, and changes in physical distancing, wearing masks, and other COVID-19 prevention behaviors (4). SARS-CoV-2 transmission occurs more easily in high schools than in elementary schools (4), and outbreaks have been associated with high school extracurricular activities (5). Vaccination of adolescents is expected to reduce the risk for COVID-19 in these settings.

Population-based COVID-19-associated hospitalization rates among adolescents were lower than were those in adults, a finding consistent with studies showing that illness is generally milder in children than in adults (G). Nevertheless, severe disease does occur, including that requiring ICU admission and invasive mechanical ventilation. Most (70.6\%) adolescents in this study whose primary reason for hospitalization was COVID-19-associated illness had at least one underlying medical condition, which is lower than the percentage of hospitalized adults with an underlying medical condition (92\%) (7). Nearly $30 \%$ of these adolescents had no reported underlying medical

\footnotetext{
I9 https://gis.cdc.gov/grasp/COVIDNet/COVID19_3.html

*** https://covid.cdc.gov/covid-data-tracker/\#variant-proportions
} 
TABLE. Demographic and clinical characteristics and outcomes among adolescents aged 12-17 years with laboratory-confirmed COVID-19associated hospitalizations, by primary reason for admission - COVID-NET, 14 states,* January 1, 2021-March 31, 2021

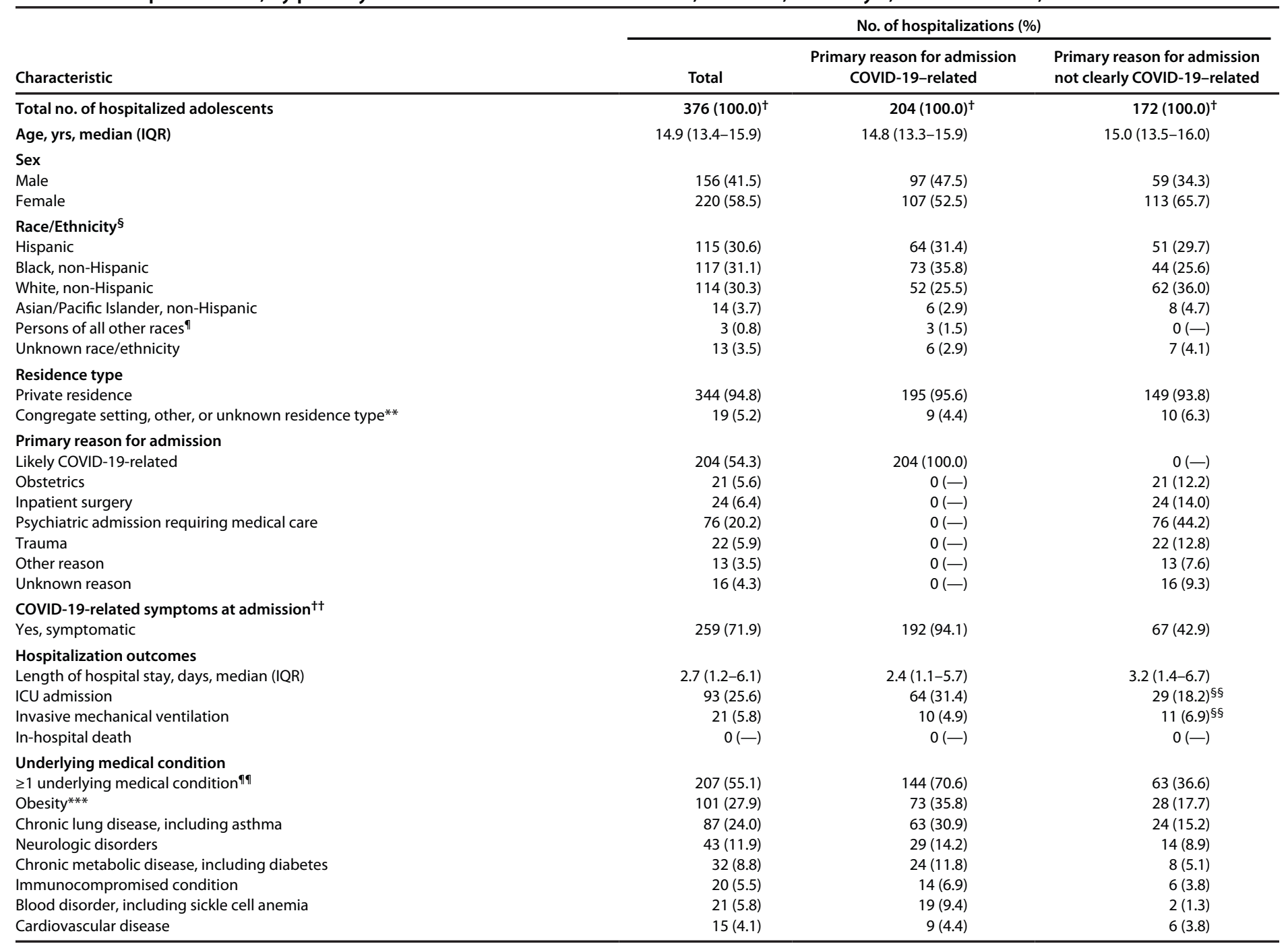

Abbreviations: COVID-NET = Coronavirus Disease 2019-Associated Hospitalization Surveillance Network; ICU = intensive care unit; IQR = interquartile range.

* Includes persons admitted to a hospital with an admission date between January 1, 2021 and March 31, 2021. Counties included in COVID-NET surveillance: California (Alameda, Contra Costa, and San Francisco counties); Colorado (Adams, Arapahoe, Denver, Douglas, and Jefferson counties); Connecticut (Middlesex and New Haven counties); Georgia (Clayton, Cobb, DeKalb, Douglas, Fulton, Gwinnett, Newton, and Rockdale counties); lowa (one county represented); Maryland (Allegany, Anne Arundel, Baltimore, Baltimore City, Calvert, Caroline, Carroll, Cecil, Charles, Dorchester, Frederick, Garrett, Harford, Howard, Kent, Montgomery, Prince George's, Queen Anne's, St. Mary's, Somerset, Talbot, Washington, Wicomico, and Worcester counties); Michigan (Clinton, Eaton, Genesee, Ingham, and Washtenaw counties); Minnesota (Anoka, Carver, Dakota, Hennepin, Ramsey, Scott, and Washington counties); New Mexico (Bernalillo, Chaves, Doña Ana, Grant, Luna, San Juan, and Santa Fe counties); New York (Albany, Columbia, Genesee, Greene, Livingston, Monroe, Montgomery, Ontario, Orleans, Rensselaer, Saratoga, Schenectady, Schoharie, Wayne, and Yates counties); Ohio (Delaware, Fairfield, Franklin, Hocking, Licking, Madison, Morrow, Perry, Pickaway and Union counties); Oregon (Clackamas, Multnomah, and Washington counties); Tennessee (Cheatham, Davidson, Dickson, Robertson, Rutherford, Sumner, Williamson, and Wilson counties); and Utah (Salt Lake County).

$\dagger$ Data are missing for $<5$ percent of observations for all variables.

$\S$ If ethnicity was unknown, non-Hispanic ethnicity was assumed.

II Includes non-Hispanic persons reported as other or multiple races.

** Unknown residence types are those which have not yet been ascertained from the medical chart but have not been determined to be missing.

t+ COVID-19-related symptoms included respiratory symptoms (congested/runny nose, cough, hemoptysis/bloody sputum, shortness of breath/respiratory distress, sore throat, upper respiratory infection, influenza-like illness, wheezing) and non-respiratory symptoms (abdominal pain, altered mental status/confusion, anosmia/decreased smell, chest pain, conjunctivitis, diarrhea, dysgeusia/decreased taste, fatigue, fever/chills, headache, muscle aches/myalgias, nausea/vomiting, rash, seizures). Symptoms are abstracted from the medical chart and might not be complete.

\$ ICU admission and invasive mechanical ventilation are not mutually exclusive categories, and patients could have received both. Of the 31 patients whose primary reason for admission was not COVID-19-related but who were admitted to the ICU or received invasive mechanical intervention, nine (29.0\%) were admitted because of trauma and 14 (45.2\%) were admitted for a psychiatric admission requiring medical care, including suicide attempts or overdoses.

9ा Defined as $\geq 1$ of the following: chronic lung disease, chronic metabolic disease, blood disorder/hemoglobinopathy, cardiovascular disease, neurologic disorder, immunocompromised condition, renal disease, gastrointestinal/liver disease, rheumatologic/autoimmune/inflammatory condition, obesity, feeding tube dependency, wheelchair dependency, or pregnancy.

*** Obesity is defined as body mass index $\left(\mathrm{kg} / \mathrm{m}^{2}\right) \geq 95$ th percentile for age and sex based on CDC growth charts among children, a discharge diagnosis of obesity indicated by International Classification of Diseases, Tenth Edition, Clinical Modification (ICD-10-CM) codes, or obesity is indicated as an underlying condition on the COVID-NET case report form for adolescents who were not pregnant at the time of admission. 
FIGURE 1. Three-week moving average COVID-19-associated hospitalization rates* among children and adolescents aged <18 years, by age group - COVID-NET, 14 states, $^{\dagger}$ March 1, 2020-April 24, 2021

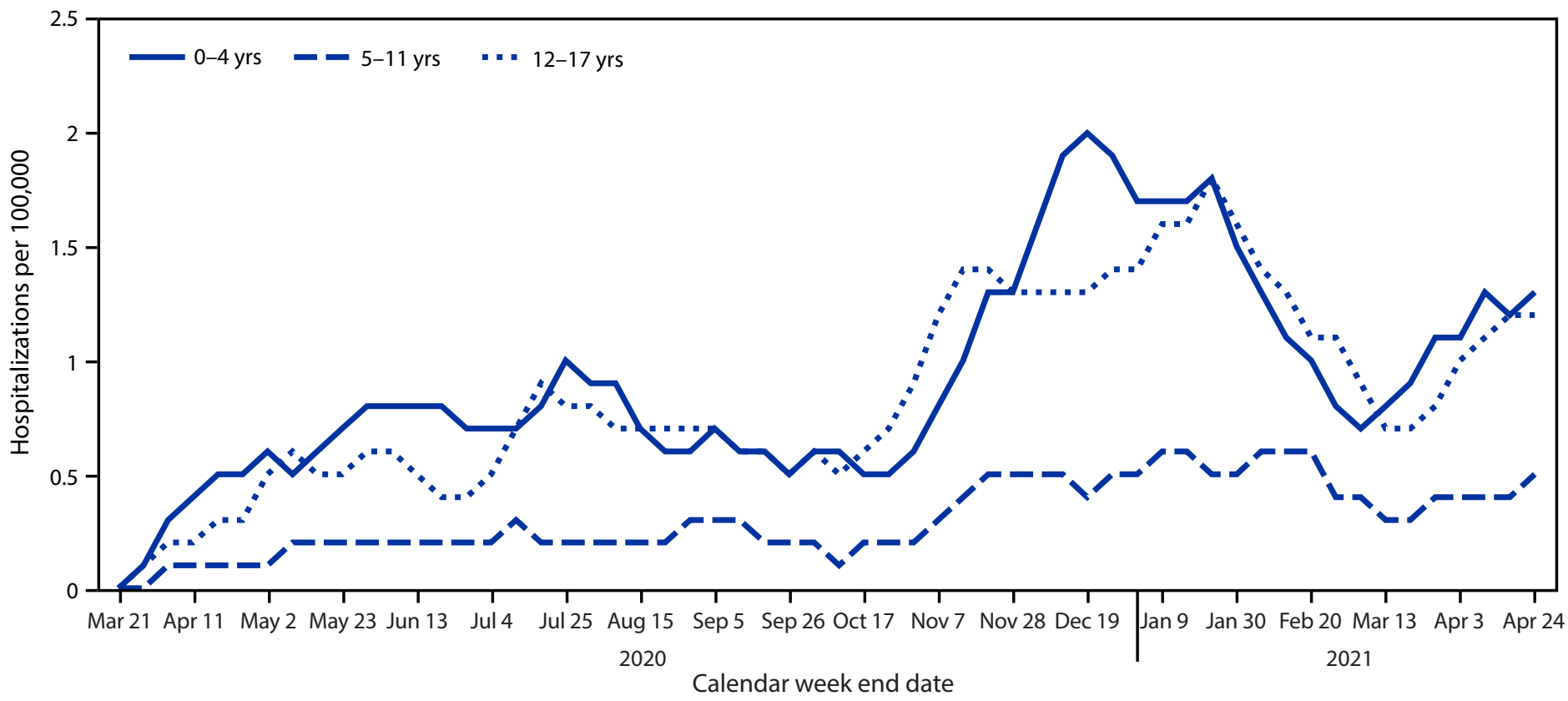

Abbreviation: COVID-NET = Coronavirus Disease 2019-Associated Hospitalization Surveillance Network.

* Number of patients with laboratory-confirmed COVID-19-associated hospitalizations per 100,000 population.

+ COVID-NET sites are in the following 14 states: California, Colorado, Connecticut, Georgia, lowa, Maryland, Michigan, Minnesota, New Mexico, New York, Ohio, Oregon, Tennessee, and Utah.

condition, indicating that healthy adolescents are also at risk for severe COVID-19-associated disease. In addition, approximately two thirds of adolescents hospitalized with COVID-19 were Hispanic or non-Hispanic Black persons, consistent with studies showing an increased incidence of COVID-19 among racial and ethnic minority populations and signifying an urgent need to ensure equitable access to vaccines for these groups (8). Vaccination is effective in preventing hospitalization among adults (9); similarly, widespread vaccination of adolescents will likely reduce COVID-19-associated hospitalizations, and potential sequelae from COVID-19 in adolescents, including multisystem inflammatory syndrome in children (MIS-C), a rare but serious complication of COVID-19 (10).

During a comparable period, adolescent hospitalization rates associated with COVID-19 exceeded those for seasonal influenza, another respiratory virus that can cause hospitalization and death in adolescents and for which a vaccine is recommended in this age group. ${ }^{\dagger \dagger}$ This widespread circulation of SARS-CoV-2 occurred despite containment measures such as school closures, wearing masks, and physical distancing, none of which had been enacted during the historical influenza seasons. Without these containment measures, the rates of COVID-19-associated hospitalization might have been substantially higher.

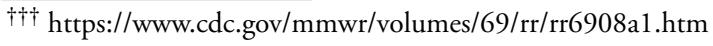

The findings in this report are subject to at least five limitations. First, the primary reason for hospital admission was not always clear, and some (45.7\%) adolescents who met the COVID-NET case definition were hospitalized for reasons that might not have been primarily related to COVID-19, despite a positive SARS-CoV-2 laboratory test result; these hospitalizations were included in rate calculations. Thus, rates of hospitalizations for COVID-19 might be overestimated. Second, laboratory confirmation depends on clinician-ordered testing and hospital testing policies for SARS-CoV-2 (COVID-NET) and influenza (FluSurv-NET); consequently, hospitalization rates might also be underestimated. Given more widespread testing for SARS-CoV-2 compared with influenza, the lack of adjustment for testing practices likely disproportionately affects influenza rates compared with COVID-19 rates. Third, adolescents hospitalized with MIS-C might not be identified if testing occurred $>14$ days before admission, potentially leading to an underestimate of severe COVID-19-associated disease. Fourth, the Pfizer-BioNTech COVID-19 vaccine had been approved for and administered to adolescents aged 16-17 years during this study period; therefore, rates of COVID-19-associated hospitalization in adolescents aged 16-17 years might differ from those in adolescents aged $12-15$ years who were not previously eligible for vaccination, and could affect the overall hospitalization rate for all adolescents. Finally, hospitalization rates are preliminary and might change as additional data are reported. 
FIGURE 2. Cumulative rates for COVID-19-associated hospitalizations* compared with influenza-associated hospitalizations ${ }^{\dagger}$ among adolescents aged 12-17 years, by surveillance week ${ }^{\S}-$ COVID-NET $^{\natural}$ and FluSurv-NET, ${ }^{* *} 14$ states, $^{+\dagger} 2017-2021^{\S \S}$

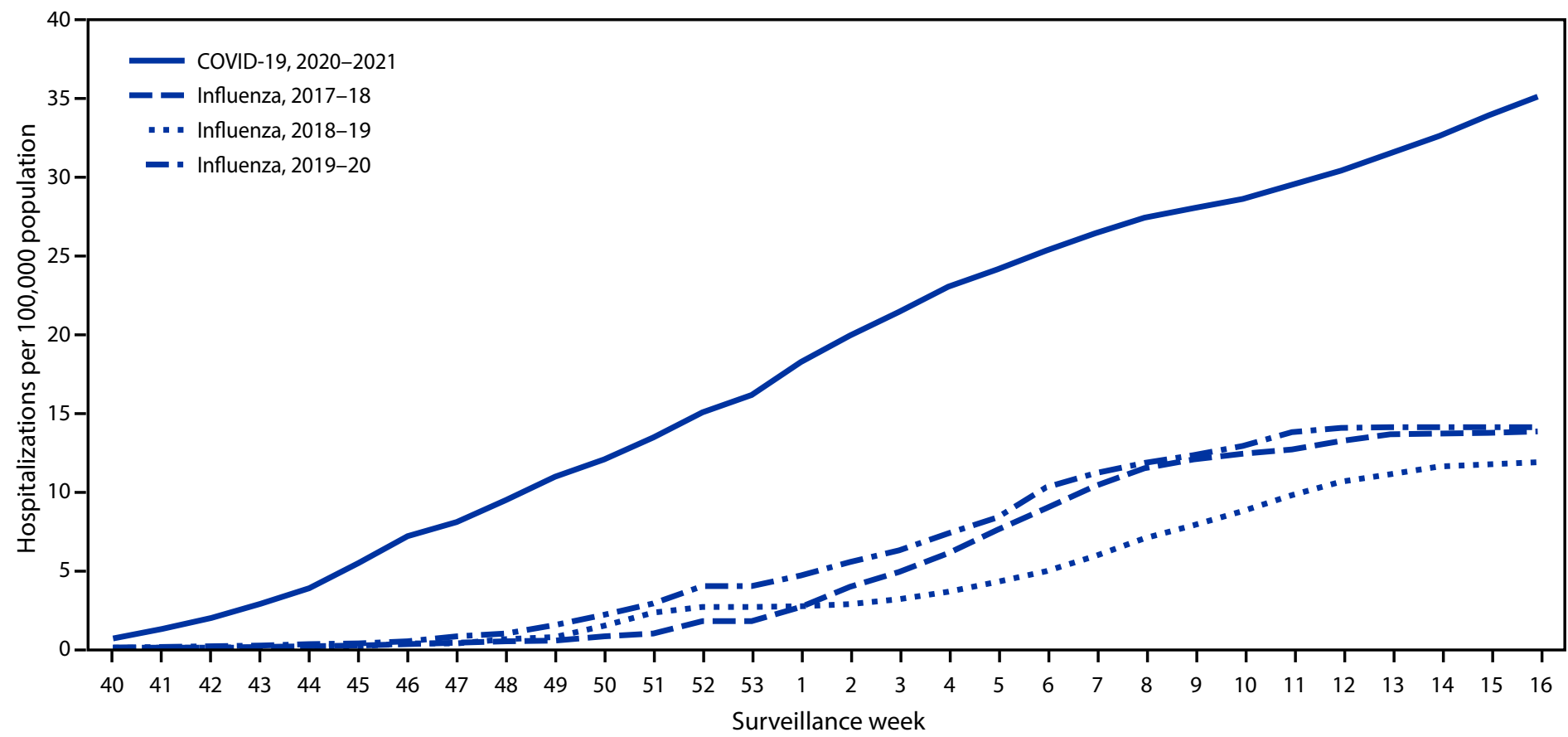

Abbreviations: COVID-NET = Coronavirus Disease 2019-Associated Hospitalization Surveillance Network; FluSurv-NET = Influenza Hospitalization Surveillance Network.

* Number of patients with laboratory-confirmed COVID-19-associated hospitalizations per 100,000 population.

† Number of patients with laboratory-confirmed influenza-associated hospitalizations per 100,000 population.

$\S$ Surveillance week is based on the epidemiologic week for disease reporting and lasts Sundays through Saturdays. MMWR week numbering is sequential beginning with 1 and incrementing with each week to a maximum of 52 or 53 . The three influenza seasons had no surveillance week 53 , so values from surveillance week 52 were imputed to surveillance week 53. https://wwwn.cdc.gov/nndss/document/MMWR_week_overview.pdf

" COVID-NET is a population-based surveillance system of laboratory-confirmed COVID-19-associated hospitalizations in 99 counties across 14 states. COVID-19associated hospitalizations among residents in a predefined surveillance catchment area who received a positive test for SARS-CoV-2 (the virus that causes COVID-19) during hospitalization or $\leq 14$ days before admission are included in surveillance.

** FluSurv-NET is a population-based surveillance system of laboratory-confirmed influenza-associated hospitalizations in 81 counties across 13 states (for the period included) and is conducted annually during October 1-April 30. Influenza-associated hospitalizations among residents in a predefined surveillance catchment area who received a positive test for influenza during hospitalization or $\leq 14$ days before admission are included in surveillance.

${ }^{+\dagger}$ COVID-NET and FluSurv-NET sites were in the following 14 states for the period shown: California, Colorado, Connecticut, Georgia, lowa (COVID-NET only), Maryland, Michigan, Minnesota, New Mexico, New York, Ohio, Oregon, Tennessee, and Utah.

$\S \S$ Cumulative COVID-19-associated hospitalization rates among adolescents aged 12-17 years during October 1, 2020-April 24, 2021, were compared with influenzaassociated hospitalization rates in the same age group during October 1-April 30 across three seasons (2017-18, 2018-19, and 2019-20) using data from FluSurv-NET.

Recent increases in COVID-19-associated hospitalization rates and the potential for severe disease requiring ICU admission, including invasive mechanical ventilation, among adolescents indicate an urgent need for vaccination in combination with correct and consistent mask wearing by persons not yet fully vaccinated or when required by laws, rules, or regulations. Highly effective COVID-19 vaccines are now available to adolescents as an additional evidence-based prevention measure (9); expansion of COVID-19 vaccination of adolescents, with particular attention to racial and ethnic minority groups disproportionately affected by severe COVID-19, is expected to reduce COVID-19-associated morbidity within this age group.

\section{Acknowledgments}

Joelle Nadel, Jeremy Roland, Brooke Heidenga, Roxanne Archer, Monica Napoles, Susan Brooks, California Emerging Infections Program;
Linda Niccolai, Connecticut Emerging Infections Program, Yale School of Public Health; Elisabeth Vaeth, Cindy Zerrlaut, David Blythe, Maryland Department of Health; Rachel Park, Michelle Wilson, Maryland Emerging Infections Program, Johns Hopkins Bloomberg School of Public Health; Jim Collins, Sam Hawkins, Justin Henderson, Shannon Johnson, Val Tellez Nunez, Sue Kim, Lauren Leegwater, Sierra Peguies-Khan, Chloe Brown, Michigan Department of Health and Human Services; Austin Bell, Kalyla Bilski, Emma Contestabile, Kristen Ehresmann, Claire Henrichsen, Emily Holodick, Lisa Nguyen, Katherine Schleiss, Samantha Siebman, Julie Hottinger, Amanda Gordon, Minnesota Department of Health; Kerianne Engesser, Suzanne McGuire, Adam Rowe, New York State Department of Health; Kathy Billings, Katie Dyer, Anise Elie, Karen Leib, Terri McMinn, Danielle Ndi, Manideepthi Pemmaraju, John Ujwok, Vanderbilt University Medical Center; Ilene Risk, Mary Hill, Salt Lake County Health Department; Keegan McCaffrey, Utah Department of Health; Mimi Huynh, Council of State and Territorial Epidemiologists; Sonja Mali Nti-Berko, Susan Conner Gantt, Alissa O'Halloran, Charisse Cummings, Rachel Holstein, Carrie Reed, CDC. 


\section{Summary}

What is already known about this topic?

Most COVID-19-associated hospitalizations occur in adults, but severe disease occurs in all age groups, including adolescents aged 12-17 years.

What is added by this report?

COVID-19 adolescent hospitalization rates from COVID-NET peaked at 2.1 per 100,000 in early January 2021 , declined to 0.6 in mid-March, and rose to 1.3 in April. Among hospitalized adolescents, nearly one third required intensive care unit admission, and $5 \%$ required invasive mechanical ventilation; no associated deaths occurred.

What are the implications for public health practice?

Recent increased hospitalization rates in spring 2021 and potential for severe disease reinforce the importance of continued COVID-19 prevention measures, including vaccination and correct and consistent mask wearing among persons not fully vaccinated or when required.

Corresponding author: Fiona P. Havers, fhavers@cdc.gov.

${ }^{1}$ CDC COVID-19 Response Team; ${ }^{2}$ California Emerging Infections Program, Oakland, California; ${ }^{3}$ Career Epidemiology Field Officer Program, CDC; ${ }^{4}$ Colorado Department of Public Health and Environment, ${ }^{5}$ Connecticut Emerging Infections Program, Yale School of Public Health, New Haven, Connecticut; ${ }^{6}$ Departments of Medicine and Pediatrics, Emory University School of Medicine, Atlanta, Georgia; ${ }^{7}$ Georgia Emerging Infections Program, Georgia Department of Public Health; ${ }^{8}$ Atlanta Veterans Affairs Medical Center, Atlanta, Georgia; ${ }^{9}$ Division of Infectious Diseases, School of Medicine, Emory University, Atlanta Georgia; ${ }^{10}$ Iowa Department of Public Health; ${ }^{11}$ Maryland Department of Health; ${ }^{12}$ Michigan Department of Health and Human Services; ${ }^{13}$ Minnesota Department of Health; ${ }^{14} \mathrm{New}$ Mexico Emerging Infections Program, University of New Mexico, Albuquerque, New Mexico; ${ }^{15}$ New Mexico Emerging Infections Program, New Mexico Department of Health; ${ }^{16} \mathrm{New}$ York State Department of Health; ${ }^{17}$ University of Rochester School of Medicine and Dentistry, Rochester, New York; ${ }^{18}$ Ohio Department of Health;

${ }^{19}$ Public Health Division, Oregon Health Authority; ${ }^{20}$ Vanderbilt University Medical Center, Nashville, Tennessee; ${ }^{21}$ Salt Lake County Health Department, Salt Lake City, Utah; ${ }^{22}$ General Dynamics Information Technology, Atlanta, Georgia; ${ }^{23}$ Influenza Division, National Center for Immunization and Respiratory Diseases, CDC.

All authors have completed and submitted the International Committee of Medical Journal Editors form for disclosure of potential conflicts of interest. Andrea George, Laurie M. Billing, Libby Reeg, Alexander Kohrman, Andrew Weigel, Kenzie Teno, and Jess Shiltz report grant funding from the Council of State and Territorial Epidemiologists. William Schaffner reports personal fees from VBI Vaccines. Evan J. Anderson reports grants for clinical trials from Pfizer, Merck, PaxVax, Micron, Sanofi-Pasteur, Janssen, MedImmune and GSK, and personal fees for consulting from SanofiPasteur, Pfizer, and Medscape, and for data safety monitoring board service from Kentucky Bioprocessing, Inc. and Sanofi-Pasteur; and funding from the National Institutes of Health to conduct clinical trials of Moderna and Janssen COVID-19 vaccines. No other potential conflicts of interest were disclosed.

\section{COVID-NET Surveillance Team}

Gretchen Rothrock, California Emerging Infections Program, Oakland, California; Arthur Reingold, University of California;
Millen Tsegaye, Colorado Department of Public Health \& Environment; Sarah McLafferty, Colorado Department of Public Health \& Environment; Amber Maslar, Connecticut Emerging Infections Program; Paula Clogher, Connecticut Emerging Infections Program; Adam Misiorski, Connecticut Emerging Infections Program; Christina Parisi, Connecticut Emerging Infections Program; Maria Correa, Connecticut Emerging Infections Program; Tessa Carter, Connecticut Emerging Infections Program; Carol Lyons, Connecticut Emerging Infections Program; Daewi Kim, Connecticut Emerging Infections Program; Gaggan Brar, Connecticut Emerging Infections Program; Emily Fawcett, Georgia Emerging Infections Program, Georgia Department of Public Health, Veterans Affairs Medical Center, Foundation for Veterans Education and Research; Allison Roebling, Georgia Emerging Infections Program, Georgia Department of Public Health, Veterans Affairs Medical Center, Foundation for Veterans Education and Research; Katelyn Ward, Georgia Emerging Infections Program, Georgia Department of Public Health, Veterans Affairs Medical Center, Foundation for Veterans Education and Research; Jana Manning, Georgia Emerging Infections Program, Georgia Department of Public Health, Veterans Affairs Medical Center, Foundation for Veterans Education and Research; Asmith Joseph, Georgia Emerging Infections Program, Georgia Department of Public Health, Veterans Affairs Medical Center, Foundation for Veterans Education and Research; Chandler Surell, Georgia Emerging Infections Program, Georgia Department of Public Health, Veterans Affairs Medical Center, Foundation for Veterans Education and Research; Daniel Pizarro, Georgia Emerging Infections Program, Georgia Department of Public Health, Veterans Affairs Medical Center, Foundation for Veterans Education and Research; Jeremiah Williams, Georgia Emerging Infections Program, Georgia Department of Public Health, Veterans Affairs Medical Center, Foundation for Veterans Education and Research; Rayna Ceaser, Georgia Emerging Infections Program, Georgia Department of Public Health, Veterans Affairs Medical Center, Foundation for Veterans Education and Research; Stephanie Lehman, Georgia Emerging Infections Program, Georgia Department of Public Health, Veterans Affairs Medical Center, Foundation for Veterans Education and Research; Taylor Eisenstein, Georgia Emerging Infections Program, Georgia Department of Public Health, Veterans Affairs Medical Center, Foundation for Veterans Education and Research; Gracie Chambers, Georgia Emerging Infections Program, Georgia Department of Public Health, Veterans Affairs Medical Center, Foundation for Veterans Education and Research; Grayson Kallas, Division of Infectious Diseases, School of Medicine, Emory University, Emerging Infections Program, Georgia Department of Health; Lauren Russell, Division of Infectious Diseases, School of Medicine, Emory University, Emerging Infections Program, Georgia Department of Health; Suzanne Segler, Division of Infectious Diseases, School of Medicine, Emory University, Emerging Infections Program, Georgia Department of Health; David Blythe, Maryland Department of Health; Alicia Brooks, Maryland Department of Health; Erica Bye, Minnesota Department of Health; Richard Danila, Minnesota Department of Health; Cory Cline, New Mexico Department of Health; Susan Ropp, New Mexico Department 
of Health; Chad Smelser, New Mexico Department of Health; Daniel Sosin, New Mexico Department of Health; Salina Torres, New Mexico Department of Health; Kathy Angeles, New Mexico Emerging Infections Program; Melissa Christian, New Mexico Emerging Infections Program; Nancy Eisenberg, New Mexico Emerging Infections Program; Kristina Flores, New Mexico Emerging Infections Program; Caroline Habrun, New Mexico Emerging Infections Program; Emily Hancock, New Mexico Emerging Infections Program; Sarah Khanlian, New Mexico Emerging Infections Program; Meaghan Novi, New Mexico Emerging Infections Program; Erin Phipps, New Mexico Emerging Infections Program; Dominic Rudin, New Mexico Emerging Infections Program; Yadira Salazar-Sanchez, New Mexico Emerging Infections Program; Judith Segall, New Mexico Emerging Infections Program; Sarah Shrum Davis, New Mexico Emerging Infections Program; Grant Barney, New York State Department of Health; Christina Felsen, University of Rochester School of Medicine and Dentistry, Rochester, New York; Sophrena Bushey, University of Rochester School of Medicine and Dentistry, Rochester, New York; Kevin Popham, University of Rochester School of Medicine and Dentistry, Rochester, New York; Virginia Cafferky, University of Rochester School of Medicine and Dentistry, Rochester, New York; Christine Long, University of Rochester School of Medicine and Dentistry, Rochester, New York; RaeAnne Kurtz, University of Rochester School of Medicine and Dentistry, Rochester, New York; Nicole West, Public Health Division, Oregon Health Authority; Ama Owusu-Dommey, Public Health Division, Oregon Health Authority; Breanna McArdle, Public Health Division, Oregon Health Authority; Emily Youngers, Public Health Division, Oregon Health Authority; Kylie Seeley, Oregon Health \& Science University School of Medicine, Portland, Oregon; Tiffanie Markus, Vanderbilt University Medical Center; Amanda Carter, Salt Lake County Health Department, Utah; Andrea Price, Salt Lake County Health Department, Utah; Andrew Haraghey, Salt Lake County Health Department, Utah; Ashley Swain, Salt Lake County Health Department, Utah; Caitlin Shaw, Salt Lake County Health Department, Utah; Ian Buchta, Salt Lake County Health Department, Utah; Jake Ortega, Salt Lake County Health Department, Utah; Laine McCullough, Salt Lake County Health Department, Utah; Ryan Chatelain, Salt Lake County Health Department, Utah; Tyler Riedesel, Salt Lake County Health Department, Utah.

\section{References}

1. Shekerdemian LS, Mahmood NR, Wolfe KK, et al.; International COVID-19 PICU Collaborative. Characteristics and outcomes of children with coronavirus disease 2019 (COVID-19) infection admitted to US and Canadian pediatric intensive care units. JAMA Pediatr 2020;174:868-73. PMID:32392288 https://doi.org/10.1001/ jamapediatrics.2020.1948

2. Kim L, Whitaker M, O'Halloran A, et al.; COVID-NET Surveillance Team. Hospitalization rates and characteristics of children aged $<18$ years hospitalized with laboratory-confirmed COVID-19-COVID-NET, 14 states, March 1-July 25, 2020. MMWR Morb Mortal Wkly Rep 2020;69:1081-8. PMID:32790664 https://doi.org/10.15585/mmwr. mm6932e3

3. Chaves SS, Lynfield R, Lindegren ML, Bresee J, Finelli L. The US influenza hospitalization surveillance network. Emerg Infect Dis 2015;21:1543-50. PMID:26291121 https://doi.org/10.3201/ eid2109.141912

4. Goldstein E, Lipsitch M, Cevik M. On the effect of age on the transmission of SARS-CoV-2 in households, schools, and the community. J Infect Dis 2021;223:362-9. PMID:33119738 https:// doi.org/10.1093/infdis/jiaa691

5. Atherstone C, Siegel M, Schmitt-Matzen E, et al. SARS-CoV-2 transmission associated with high school wrestling tournamentsFlorida, December 2020-January 2021. MMWR Morb Mortal Wkly Rep 2021;70:141-3. PMID:33507895 https://doi.org/10.15585/ mmwr.mm7004e4

6. Davies NG, Klepac P, Liu Y, Prem K, Jit M, Eggo RM; CMMID COVID-19 working group. Age-dependent effects in the transmission and control of COVID-19 epidemics. Nat Med 2020;26:1205-11. PMID:32546824 https://doi.org/10.1038/s41591-020-0962-9

7. Kim L, Garg S, O'Halloran A, et al. Risk factors for intensive care unit admission and in-hospital mortality among hospitalized adults identified through the U.S. Coronavirus Disease 2019 (COVID-19)-Associated Hospitalization Surveillance Network (COVID-NET). Clin Infect Dis 2021;72:e206-14. PMID:32674114 https://doi.org/10.1093/cid/ciaa1012

8. Mackey K, Ayers CK, Kondo KK, et al. Racial and ethnic disparities in COVID-19-related infections, hospitalizations, and deaths: a systematic review. Ann Intern Med 2021;174:362-73. PMID:33253040 https:// doi.org/10.7326/M20-6306

9. Tenforde MW, Olson SM, Self WH, et al.; IVY Network; HAIVEN Investigators. Effectiveness of Pfizer-BioNTech and Moderna vaccines against COVID-19 among hospitalized adults aged $\geq 65$ years-United States, January-March 2021. MMWR Morb Mortal Wkly Rep 2021;70:674-9. PMID:33956782 https://doi.org/10.15585/mmwr. $\mathrm{mm} 7018 \mathrm{e} 1$

10. Feldstein LR, Rose EB, Horwitz SM, et al.; Overcoming COVID-19 Investigators; CDC COVID-19 Response Team. Multisystem inflammatory syndrome in U.S. children and adolescents. N Engl J Med 2020;383:334-46. PMID:32598831 https://doi.org/10.1056/ NEJMoa2021680 